\title{
E2f3b plays an essential role in myogenic differentiation through isoform-specific gene regulation
}

\author{
Patrik Asp, Diego Acosta-Alvear, Mary Tsikitis, Chris van Oevelen, and Brian David Dynlacht ${ }^{1}$ \\ New York University School of Medicine, New York University Cancer Institute, New York, New York 10016, USA
}

\begin{abstract}
Current models posit that E2F transcription factors can be divided into members that either activate or repress transcription, in part through collaboration with the retinoblastoma $(\mathbf{p R b})$ tumor suppressor family. The E2f3 locus encodes E2f3a and E2f3b proteins, and available data suggest that they regulate cell cycle-dependent gene expression through opposing transcriptional activating and repressing activities in growing and quiescent cells, respectively. However, the role, if any, of E2F proteins, and in particular E2f3, in myogenic differentiation is not well understood. Here, we dissect the contributions of E2f3 isoforms and other activating and repressing E2Fs to cell cycle exit and differentiation by performing genome-wide identification of isoform-specific targets. We show that E2f3a and E2f3b target genes are involved in cell growth, lipid metabolism, and differentiation in an isoformspecific manner. Remarkably, using gene silencing, we show that E2f3b, but not E2f3a or other E2F family members, is required for myogenic differentiation, and that this requirement for E2f3b does not depend on pRb. Our functional studies indicate that E2f3b specifically attenuates expression of genes required to promote differentiation. These data suggest how diverse E2F isoforms encoded by a single locus can play opposing roles in cell cycle exit and differentiation.
\end{abstract}

[Keywords: E2F; E2f3b; cell cycle; ChIP-on-chip; myogenic differentiation]

Supplemental material is available at http://www.genesdev.org.

Received August 14, 2008; revised version accepted November 17, 2008.

The decision to differentiate or proliferate represents a critical point in the development of multicellular organisms. Mammalian cells must cease to proliferate and enter the $G_{0}$ phase of the cell cycle either right before or during differentiation, and this terminal cell cycle exit is regulated by a complex network of extra- and intracellular signaling pathways and checkpoints. The retinoblastoma tumor suppressor family (pRb, p107, and p130), also known as pocket proteins, act as transcriptional regulators in concert with E2F to control cell cycle entry and progression (Ren et al. 2002; Cam and Dynlacht 2003; Stevens and La Thangue 2003). It is well established that the $\mathrm{pRb}$ and E2F proteins, as well as their Drosophila homologs, also regulate genes involved in DNA repair, chromatin structure, $\mathrm{G}_{2} \mathrm{M}$ checkpoints, and differentiation (Ren et al. 2002; Dimova et al. 2003; Zhu 2005).

Pocket proteins regulate E2F activity by binding to the trans-activation domain of E2F (Sherr 2004; Giacinti and Giordano 2006). Formation of pocket protein/E2F complexes on the promoter leads to both passive and active repression of E2F target genes, resulting from interactions that both physically block the trans-activation domain of

${ }^{1}$ Corresponding author.

E-MAIL brian.dynlacht@med.nyu.edu; FAX (212) 263-6157.

Article is online at http://www.genesdev.org/cgi/doi/10.1101/gad.1727309.
E2F and promote recruitment of chromatin modifying enzymes, such as HDACs. Thus, pocket proteins function by modifying chromatin, leading to subsequent changes in expression (De Falco et al. 2006; Macaluso et al. 2006).

The E2F protein family consists of eight members, E2F1-8, and their heterodimeric DNA-binding partners, DP1 and DP2. They have been classified into activators and repressors, depending on the transcriptional response when bound to promoters. E2F1-3 are generally regarded as activators, while E2F4-6 are repressors (Blais and Dynlacht 2007). E2F7-8 were identified recently and are less well characterized, although their activities appear to be primarily inhibitory (Moon and Dyson 2008). E2Fs and pocket proteins exhibit tissue and developmental stage specificity. For example, E2F2 has been shown to play a role in the hematopoietic compartment (Cloud et al. 2002; Dirlam et al. 2007). Recently, E2f3 has been indirectly implicated in the maintenance of trophoblast stem cells in the placenta and has been shown to play a role in neuronal migration (McClellan et al. 2007; Wenzel et al. 2007). In addition, E2f3 ${ }^{-/-}$mice die of congestive heart failure, suggesting a defect in heart muscle development (Cloud et al. 2002). In contrast to heart development, the role of E2Fs, if any, in skeletal muscle differentiation, has not been thoroughly explored. 
The mouse E2f3 locus gives rise to two transcripts encoding longer (E2f3a) and shorter (E2f3b) isoforms that are distinguished exclusively by 122 and six unique Nterminal residues, respectively. Both isoforms possess identical DNA-binding, dimerization, and trans-activation domains that allow binding of heterodimeric partners and pocket proteins, facilitating recruitment to canonical E2F target genes. The two isoforms are highly related, thus explaining their biological redundancy $(\mathrm{He}$ et al. 2000; Leone et al. 2000; Danielian et al. 2008; Tsai et al. 2008). However, the two proteins do not show complete functional overlap. For example, E2f3a is predominantly expressed in growing cells, with enrichment in $\mathrm{S}$ phase, while E2f3b is expressed throughout the cell cycle and in quiescent cells (Leone et al. 2000). Further, E2f3a has been shown to be essential for proper neural development in the mouse retina, while E2f3b has been shown to play a role in oncogene surveillance via transcriptional repression of the Arf locus (Aslanian et al. 2004; Chen et al. 2007). It has been demonstrated recently that E2f3a alone is sufficient for embryonic and postnatal development in the absence of E2f1, E2f2, and E2f3b (Tsai et al. 2008). Interestingly, the same is also true when expressing E2f1 or E2f3b from the E2f3a locus. Clearly, the ability to drive embryonic and postnatal development is not a unique property of the E2f3a protein itself but is a result of spatial and temporal expression mediated by the E2f3a promoter, which could explain the apparent tissue-specific roles for different E2Fs.

Despite these findings, it is clear that E2F family members exhibit considerable redundancy, and knockout studies are complicated by the occurrence of functional compensation that can occur during development, making it difficult to elucidate the contribution of individual isoforms. Thus, distinct roles for the E2f3 isoforms have not been thoroughly investigated. Here, we took advantage of isoform-specific target identification and acute gene ablation to uncover novel, isoform-specific functions for E2f3.

We show that E2f3b plays a unique and essential role in myogenic differentiation, distinguishing it from E2f1, E2f2, and E2f3a, which are dispensable. Furthermore, loss of E2f4, another repressor E2F, has a considerably lesspronounced effect. Genome-wide identification of isoform-specific E2f3 target genes suggests that E2f3a plays a role in activating genes involved in cell cycle progression. In sharp contrast, E2f3b is recruited to genes involved in differentiation and development, suggesting a role in these processes. We also find that E2F3, especially E2f3b, binds to an unexpectedly large number of lipid metabolism genes. Surprisingly, loss of E2f3 has no overt effect on the expression of cell cycle genes, but it has a pronounced impact on E2f3b-specific, differentiation-associated genes. Unexpectedly, our data reveal that recruitment of E2f3a and E2f3b isoforms to promoters can be restored through ectopic expression, promoting transcriptional regulation of target genes in a remarkably isoform-specific manner. In some instances, E2 $\mathrm{f} 3 \mathrm{a}$ is inappropriately recruited to E2 $\mathrm{f} 3 \mathrm{~b}$ targets upon enforced expression, although it is incapable of regulating these genes, suggesting an additional layer of control subsequent to promoter recruitment. Interestingly, the role of $\mathrm{E} 2 \mathrm{f} 3 \mathrm{~b}$ in myogenic differentiation and gene-repression is $\mathrm{pRb}$-independent. Thus, we report the novel finding that E2f3 isoforms can discriminate between specific target genes, and we implicate E2f3b as a unique E2F family member that plays an essential role in mammalian differentiation and development.

\section{Results \\ E2f3 is critical for myogenic differentiation}

We sought to determine the role, if any, of E2f1, E2f2, E2f3, and E2f4 in myogenic differentiation. Expression of $\mathrm{E} 2 \mathrm{f1}$ and E2f3a was strongly down-regulated during this process, reaching barely detectable levels in mature myotubes, while E2f2, E2f3b, and E2f4 were maintained at constant levels or were slightly up-regulated (Fig. 1A). We next investigated the contribution of individual E2F proteins to myogenic differentiation by suppressing their expression using RNAi. We infected growing C2C12 myoblasts with retroviruses expressing microRNAs (miRNA) (Dickins et al. 2005; Silva et al. 2005) engineered to specifically target E2f1, E2f2, both isoforms of E2f3, and E2f4, and showed that each miRNA specifically and potently ablated expression of each individual protein. As a control, we expressed a miRNA targeting luciferase (Fig. 1B). We confirmed that miRNA-mediated protein ablation remained constant throughout differentiation by inducing differentiation and analyzing samples after 96 $h$, at which point myogenic differentiation was essentially complete (Fig. 1B). In contrast to studies in knockout mouse embryonic fibroblasts (MEFs) (Kong et al. 2007), acute knockdown did not induce strong compensatory changes in E2F protein levels, enabling us to study the contribution of each individual E2F to the process of muscle differentiation (Fig. 1B).

Next, miRNA-transduced cells were induced to differentiate and myogenic differentiation was examined by detecting the expression of myosin heavy chain (MHC), a differentiation marker (Fig. 1C). Expression of the control miRNA did not affect differentiation as compared with untreated cells (Fig. 1C). Depletion of E2f1 or E2f2 had no discernible effect on differentiation, as infected myoblasts expressing either miRNA fused to form highly organized and elongated myotubes comparable with nontransduced and control cells (Fig. 1C). In sharp contrast, depletion of E2f3 resulted in substantial inhibition of myogenic differentiation (Fig. 1C). The MHC-positive cells that arose during differentiation resembled short, stunted myotubes and were either mononucleated or consisted of few nuclei (as compared with normal myotubes) (Fig. 1C). A second, independent E2F3-directed miRNA gave indistinguishable results (data not shown).

Depletion of E2f4 also adversely affected the normal differentiation program and gave rise to a less-dense layer of myotubes compared with control cells. However, the phenotype was clearly more subtle than the one provoked by E2f3 deficiency, since E2f4-deficient myotubes were 

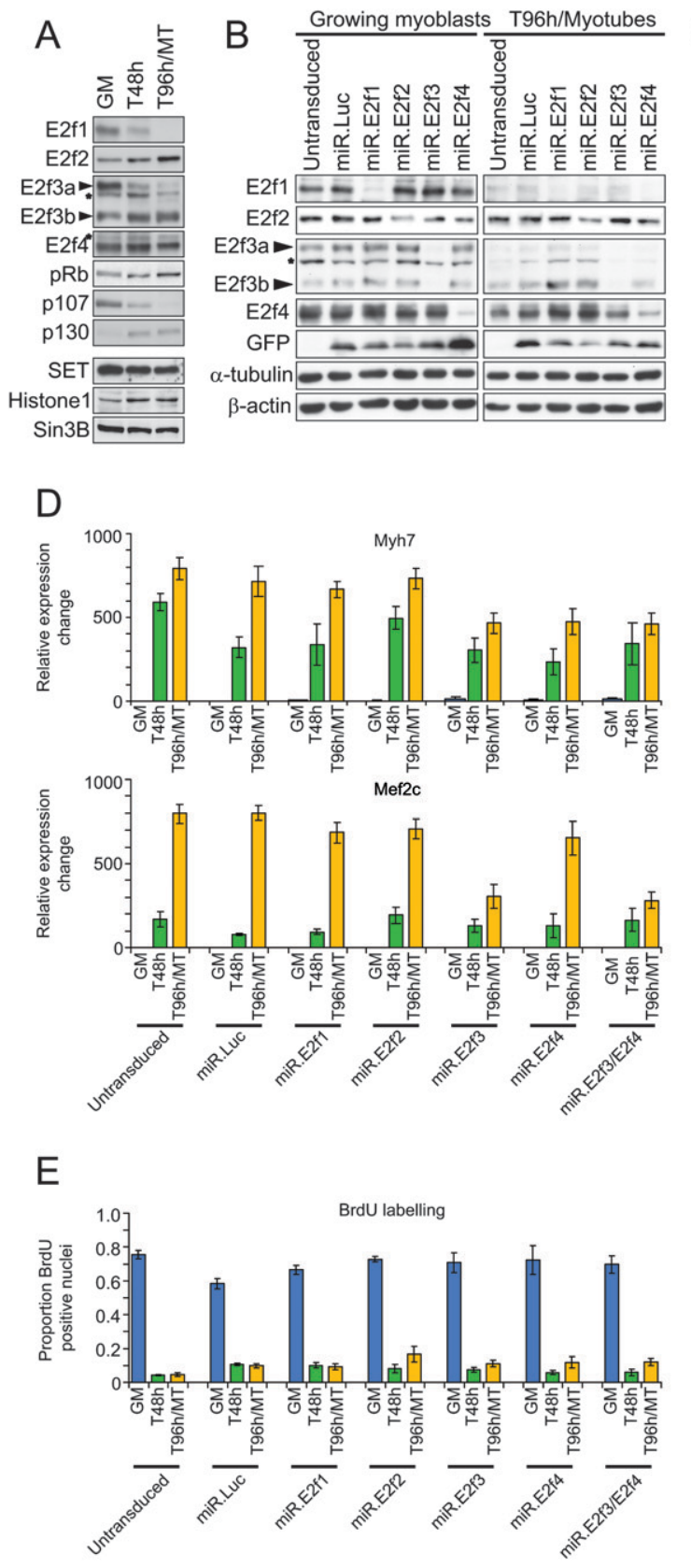
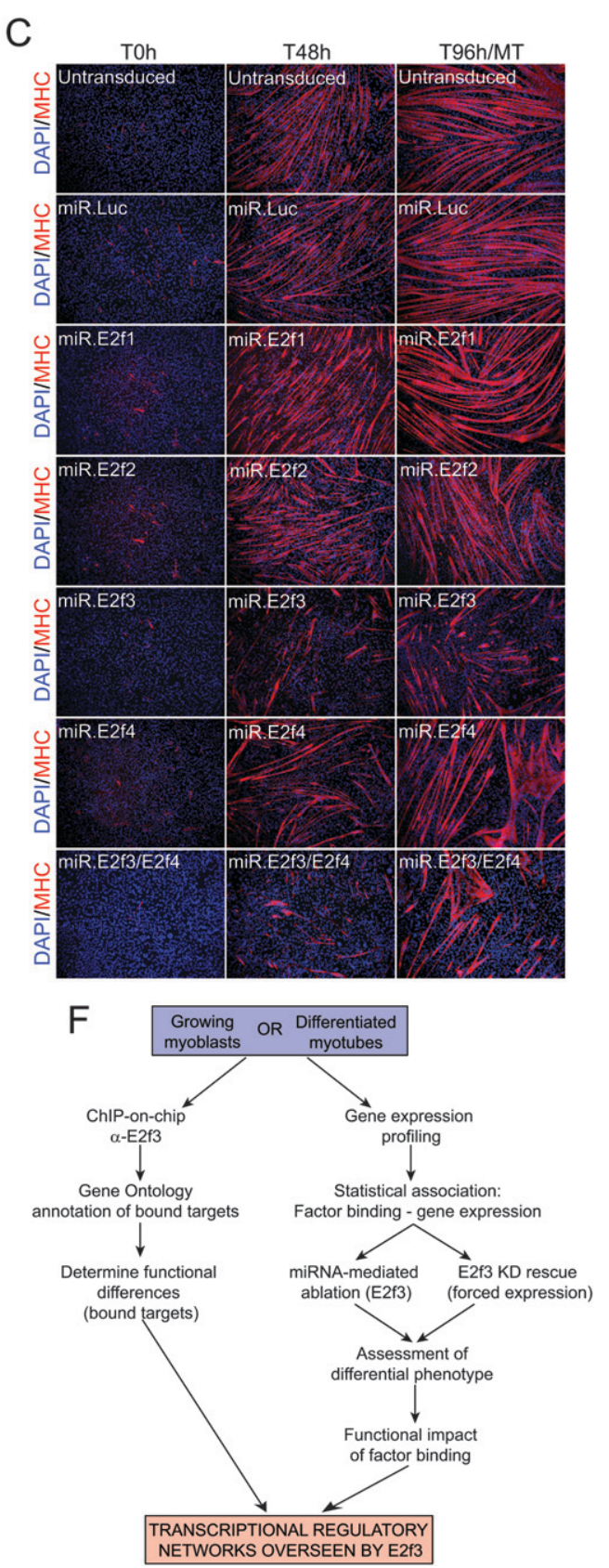

Figure 1. Expression of E2F family members during myogenesis and their role in differentiation. $(A)$ Expression of E2F and pocket protein family members during myogenic differentiation. Each protein (indicated on the left) was detected by Western blot analysis in whole-cell lysates of myoblasts and of myotubes differentiated for 48 or $96 \mathrm{~h}$ (T48h and T96h/MT, respectively). Myotubes were isolated at $96 \mathrm{~h}$. (B) Each E2F family member was ablated using miRNAs as indicated at the top. Green fluorescent protein (GFP) was coexpressed with each miRNA and serves as an expression marker. Loading control: $\beta$-actin and $\alpha$-tubulin. $\left({ }^{\star}\right)$ Nonspecific bands. $(C)$ After ablation of the indicated E2F family members, cells were induced to differentiate for 48 and $96 \mathrm{~h}$ and stained with MHC and DAPI. $(D)$ RT-qPCR analysis of the relative expression levels of the differentiation marker genes Myh7 and Mef2c in the miRNA-depletion experiment shown in $C$. Error bars depict the standard error of the mean (SEM) derived from three independent experiments. $P$-values were derived from a one-tail paired $t$-test between miR.Luc and miR.pan-E2F3-transduced cells. (E) Cells ablated for E2F family members were stained for BrdU incorporation at the indicated time points (as in $C$ ). The proportion of BrdU-positive nuclei is shown for each population. $(F)$ An outline for dissecting E2F3 function during myogenic differentiation. 
well organized and multinucleated, consisting of a nearly normal complement of nuclei (Fig. 1C). We also saw a significant increase in the number of larger, hypertrophic myotubes among the E2f4-depleted population (Fig. 1C). Simultaneous depletion of both E2f3 and E2f4 provoked a differentiation defect qualitatively similar to the one observed for E2f3 alone, and further exacerbation of the E2f3 ablation phenotype was not seen (Fig. 1C). This suggested that E2f4 did not additively contribute to the function of E2f3 in myogenic differentiation within the scope of this analysis. In addition, we surveyed the expression of two other myogenic differentiation marker genes, Myh7 and Mef2c, which were strongly up-regulated during myogenesis. We found that depletion of E2f3, E2f4, or both, but not E2f1 or E2f2, led to significantly lower expression levels of both genes, in agreement with other differentiation markers and mirroring the observed differentiation defect (Fig. 1D).

We next initiated efforts to determine the mechanism of the differentiation defect. The phenotypes observed after depleting E2f3 (or E2f4) were not a result of variations in cell density at the time of induction of differentiation, as the densities were the same for each population as determined by DAPI staining (data not shown). Furthermore, BrdU incorporation assays did not reveal aberrant DNA replication upon induction of differentiation, indicating that cells were indeed able to exit the cell cycle, despite loss of E2f3 or E2f4 (Fig. 1E). We did not detect changes in cell cycle profiles of growing myoblasts using FACS analysis, nor did we observe changes in myoblast growth rates (Supplemental Fig. $\mathrm{S} 1 \mathrm{~A}, \mathrm{~B})$. We conclude that E2f3, but not E2f1 or E2f2, is essential for proper myogenic differentiation in a manner independent of E2f4. Further, the phenotype observed upon E2f3 ablation appears to be a result of impaired differentiation rather than from a failure to exit the cell cycle.

\section{Identification of E2f3 isoform-specific target genes}

The foregoing experiments did not address whether E2f3a, E2f3b, or both, play a role in myogenic differentiation. Thus, we sought to dissect the role of each E2f3 protein in myogenic differentiation by employing a twopronged genomic strategy (Fig. 1F). Chromatin immunoprecipitation (ChIP)-on-chip analysis was used to identify E2f3 target genes and, if possible, identify isoform-specific target genes. A combination of miRNA knockdown, expression profiling, and isoform-specific rescue experiments was used to investigate specific contributions to gene transcription and differentiation. We then integrated the results of these two approaches and dissected the role of E2f3a and E2f3b in muscle differentiation.

We developed a subtractive method to identify isoformspecific E2f3 target genes by genome-wide ChIP-on-chip analysis using chromatin from growing myoblasts and mature myotubes (Fig. 2A). We made use of mouse promoter DNA microarrays that span a region from -5.5 to $+2 \mathrm{~kb}$ relative to the transcriptional start site (TSS) of $\sim 17,000$ known mouse genes. Two different antibodies against E2f3 were used, including a polyclonal antibody that recognizes a region close to the $\mathrm{C}$ terminus, which is common to both E2f3a and E2f3b (Fig. 2A). Henceforth, we will refer to this antibody and the resulting targets with the $\mathrm{E} 2 \mathrm{f} 3 \mathrm{a} / \mathrm{b}$ descriptor. We also used a monoclonal antibody that specifically recognizes the unique $\mathrm{N}$-terminal portion of $\mathrm{E} 2 \mathrm{f} 3 \mathrm{a}$, and we refer to this antibody and resulting targets with the E2f3a designation (Fig. 2A). We confirmed the specificity of each of these antibodies by ChIP using E2f3-null 3T3 cells (Supplemental Fig. S1C). Expression of E2f3a was strongly down-regulated during differentiation and was virtually undetectable in mature myotubes, while E2f3b expression persists and was slightly up-regulated in myotubes (Fig. 1A). This result allowed us to design a double-subtraction approach, wherein we identified isoform-specific target genes by (1) subtracting the gene lists generated by individual antibodies (E2f3a and E2f3ab), and (2) by comparing myoblast versus myotube populations, as the disappearance of E2f3a in myotubes allowed for the exclusive identification of E2f3b target genes (Fig. 2B). Although there are low, residual levels of E2f3a in myotubes, we do not believe that it is associated with chromatin for two reasons. Firstly, immunoprecipitation of nuclear extracts with both the E2f3a-specific and pan-E2f3 antibodies failed to show significant enrichment of E2f3a in myotubes (Fig. 2B). Secondly, residual E2f3a was not detectable at promoters that were otherwise bound by this factor in myoblasts, as determined by conventional ChIP and ChIP-on-chip (Fig. 3; Supplemental Fig. S3).

Our differential ChIP-on-chip analysis identified 471 E2f3a targets and 1374 targets of E2f3a/b in growing cells. In myotubes, E2f3a yielded no verifiable target genes, while E2f3ab identified 1607 target genes (Supplemental Table S1). Binding was observed primarily in promoter regions upstream of the TSS (E2f3a myoblasts, 84\%; E2f3ab myoblasts,73\%; and E2f3ab myotubes, 70\%) (Supplemental Table S1). Surprisingly, however, we also detected significant E2f3 binding up to $1 \mathrm{~kb}$ downstream from the TSS on several genes, especially for E2f3b, which was confirmed by conventional ChIP /Chek $1+600$ base pairs [bp], Mcm $4+75$ bp, Tmpo + 900 bp, and Rrm $2+600$ bp) (Fig. 3; Supplemental Fig. S3).

The overlap between E2f3ab and E2f3a targets in myoblasts was lower than expected (Fig. 2C). We ascribe this to variation in target enrichment stemming from the use of two antibodies with probable differences in target accessibility and epitope affinity and our use of very stringent cut-offs in our microarray analysis. This approach resulted in low false positive rates but excludes a nonnegligible number of genes for which signal intensity and binding ratios were at the lower end of the scale (Supplemental Fig. S1D). To assess the accuracy of the identification of isoform-specific targets, we screened a large number of E2f3-bound genes, as well as a selection of genes that did not display E2f3 binding using conventional ChIP (Fig. 3; Supplemental Fig. S3). Based on these results, we conclude that the accuracy of our E2f3 ChIPon-chip analyses was comparable with that of other studies of mammalian transcription factors using the same technique (Supplemental Table S1; Lee et al. 2006). 
A
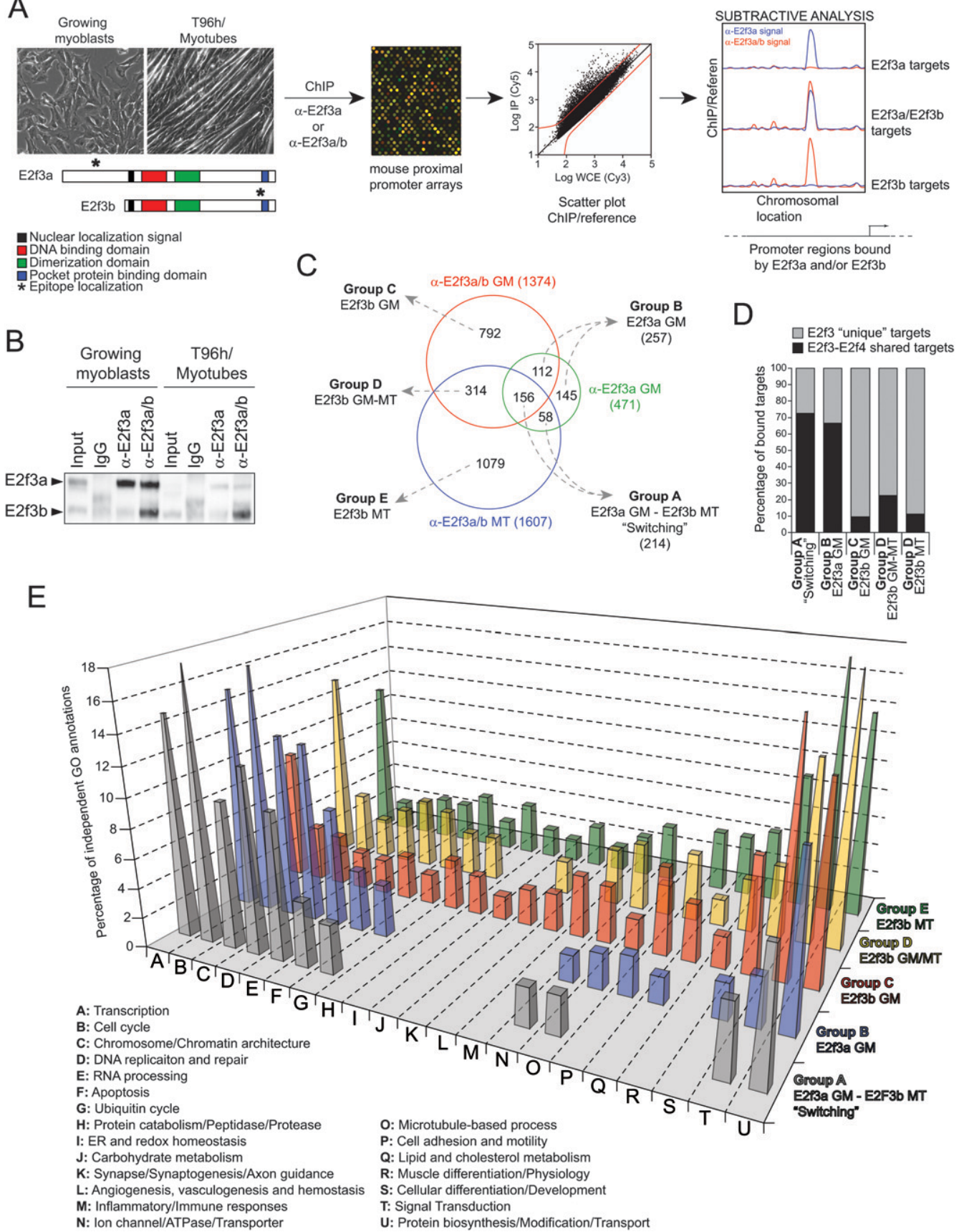

(214)

Figure 2. Identification of E2f3a and E2f3b targets. (A) Strategy for genome-wide discovery E2F3 targets and the epitopes of the two E2F3 antibodies used. (B) Enrichment test of E2F3 isoforms. (Top) Nuclear extracts from myoblasts and myotubes were immunoprecipitated with the indicated antibodies and detected by Western blotting with antibodies against E2f3a/b. $(C)$ Venn diagrams indicating the intersection between target genes in growing myoblasts and myotubes (see the text for details). Isoform-specific E2F3 target groups are labeled A-E. $(D)$ Analysis of the percent overlap between E2F3 and E2F4 targets in myotubes expressing the percentage of bound targets as a function of E2F4 target genes bound by E2F3 in each group. Corresponding Venn diagrams are shown in Supplemental Fig S3. (E) Gene Ontology (GO) analysis of E2F3 target genes expressed as the percentage of the total number of independent GO annotations within each group. Groups are indicated on the right, and GO categories on the bottom.

The subtractive analysis of differences in ChIP-on-chip, as outlined above, together with confirmatory ChIP experiments, revealed the existence of five distinct categories of E2f3 target genes (Fig. 2C). Current models for $\mathrm{E} 2 \mathrm{f} 3$ function posit that E2f3a is a transcriptional activa- tor, while E2f3b acts as a transcriptional repressor in quiescent and differentiated cells, wherein E2f3a is not expressed and E2f3b interacts with $\mathrm{pRb}$ (Kong et al. 2007). We verified that this is also true in muscle and showed that $\mathrm{E} 2 \mathrm{f} 3 \mathrm{~b}$, but not $\mathrm{E} 2 \mathrm{f} 3 \mathrm{a}$, interacts with $\mathrm{pRb}$ in fully 
Asp et al.

Figure 3. Verification of isoform-specific targets by conventional ChIP. qPCR analysis of a select number of genes representing the five E2F3 isoform-specific gene categories. Enrichment $>0.05$ is significant. Error bars depict the standard error of the mean (SEM) derived from three independent experiments.
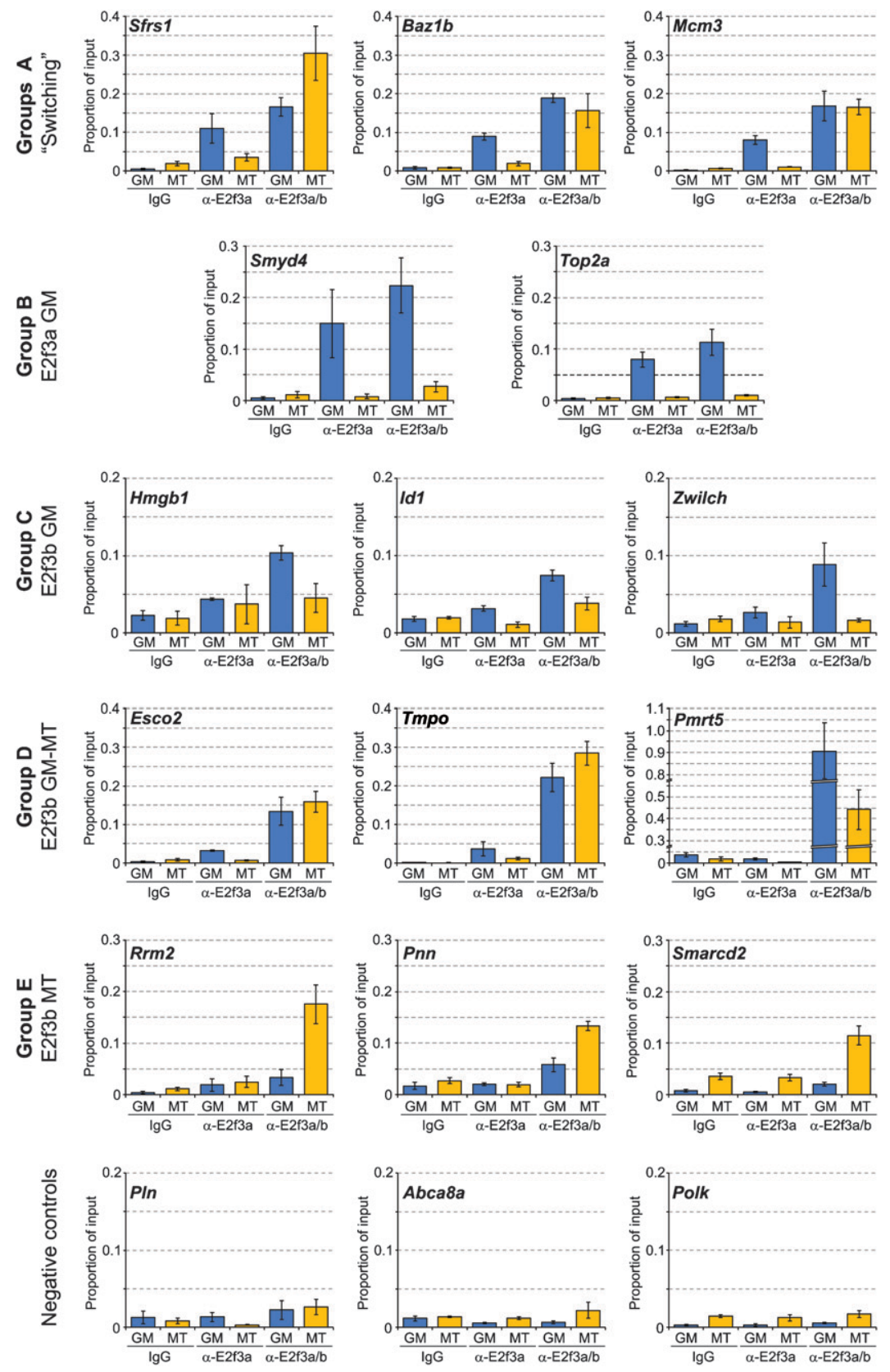

differentiated myotubes (Supplemental Fig. S5A). As pRb has a well-established role in terminal cell cycle exit and silencing of cell cycle genes, some E2f3-regulated genes might therefore be expected to undergo a transition in which E2f3a is recruited to promoters in growing myoblasts, whereas E2f3b would be recruited to the same genes in myotubes, concomitant with gene repression. Indeed, when we compared the list of E2f3a targets in myoblasts with the list of E2f3b targets in myotubes, we found 214 genes that belonged to this "switching" category, designated as group A (Fig. 2C; Supplemental Table $\mathrm{S} 2)$. We confirmed this E2f3a to E2f3b switching on several genes by conventional ChIP analysis (Fig. 3; Supplemental
S3). We also identified a set of genes exclusively bound by E2f3a in growing myoblasts, referred to as group B genes, which did not recruit E2f3b in myotubes (Fig. 2C; Supplemental Table S2). Confirmatory ChIP analysis verified that these genes were indeed E2f3a-specific (Smyd4 and Top2a in Fig. 3; Supplemental Fig. S3).

We applied the subtractive approach described above and were able to identify E2f3b-specific targets in both myoblasts and myotubes. E2f3a target genes (in myoblasts) were subtracted from those bound by $\mathrm{E} 2 \mathrm{f} 3 \mathrm{a} / \mathrm{b}$ in either condition, allowing enrichment of E2f3b-specific target genes. We then compared the lists of presumptive $\mathrm{E} 2 \mathrm{f} 3 \mathrm{~b}$ targets in myoblasts and myotubes and identified 
792 genes uniquely bound by E2f3b in myoblasts, designated group C. We observed 314 genes that were constitutively bound by E2f3b in both conditions, which we designated as group D. Lastly, we identified 1079 genes that were myotube-specific targets of E2 $\mathrm{f} 3 \mathrm{~b}$, designated as group E (Fig. 2C; Supplemental Table S2). To validate the microarray data, we analyzed a select number of genes from each group by conventional ChIP, which confirmed them to be (1) E2f3b-specific and (2) bound by E2f3b in a condition-specific manner (myoblasts and/or myotubes) (Fig. 3;Supplemental S3).

Given the pervasive role of E2f4 recruitment to cell cycle genes in differentiated myotubes (Blais et al. 2007), we also identified E2f4 targets in myotubes by performing ChIP-on-chip using identical promoter arrays (van Oevelen et al. 2008). We then compared the list of targets bound by E2f4 in myotubes with each of the five classes of E2f3 targets described above. Interestingly, we found a striking overlap between E2f4 and E2f3a target genes in groups $\mathrm{A}$ and $\mathrm{B}$. In sharp contrast, the absolute number and percentage of E2f3b-specific targets in groups $\mathrm{C}-\mathrm{E}$ that were commonly bound by E2f4 was significantly lower (Fig. 2D; Supplemental Fig S2).

\section{Functional classification of E2f3 target genes}

The identification of E2F3 isoform-specific target genes suggested that E2f3a and E2f3b have distinct biological functions. We analyzed the genes in each of the five groups by clustering them according to Gene Ontology (GO) using NIH-DAVID and extensive manual curation (Fig. 2E; Supplemental Table S2). We found that genes in groups A (switching group) and group B (E2f3a bound in myoblasts only) exhibited very similar GO profiles and were enriched in genes involved in growth and cell cycle control, transcription, RNA processing, and chromosome structure (Fig. 2E), suggesting that E2f3a is primarily involved in cell growth control.

Interestingly, the E2f3b-specific categories (groups C-E) contained significantly fewer genes involved in cell cycle control and proliferation (Fig. 2E), and group E (E2f3b bound in myotubes) was particularly depleted of such genes. Instead, these groups were very similar to each other and were enriched for several unique functional categories (such as ER redox and homeostasis, synapse/ synaptogenesis, and axon guidance) that were not represented within groups A and B (Fig. 2E). Of special interest, we identified a number of genes bound by E2f3b involved in cellular differentiation/development, and muscle differentiation/physiology (Fig. 2E), specifically linking E2f3b, but not E2f3a, to the differentiation defect observed when depleting E2f3 (Fig. 1C). We also noted that groups C-E contained genes involved in lipid/cholesterol metabolism, although with a small number of such genes also present in group B (Fig. 2E).

With our subtractive approach, we were able to clearly identify and verify E2f3a- and E2f3b-specific genes, and by clustering these genes into functional groups, a picture emerges in which E2f3a is associated with cell cycle and proliferation, while E2f3b-specific genes are primarily associated with differentiation and development. The processes of proliferation and differentiation are intimately linked, and our data places the two E2f3 isoforms at the center of fundamental crossroads in myogenic development.

\section{Correlation between E2f3 binding and gene expression}

Transcription factor binding does not necessarily predict gene regulation, and therefore, we sought to correlate E2f3 isoform binding with expression by merging our factor-binding data with gene expression profiles obtained from growing and differentiated C2C12 myoblasts (Blais et al. 2005). Data were presented as the ratio between expression levels in myotubes and growing myoblasts (MT/GM) in order to correlate E2f3 binding with dynamic changes in gene expression during myogenesis.

We found that groups $\mathrm{A}$ and $\mathrm{B}$ genes were strongly repressed during differentiation (Fig. 4A,B) in agreement with the fact that these groups primarily consisted of cell cycle- and proliferation-associated genes such as Aurkb, Cdc6, and Uhr, which were strongly down-regulated during differentiation (Supplemental Fig. S4A). The hypergeometric distribution test confirmed these correlations to be statistically significant $\left(P<10^{-10}\right)$ (Fig. 4,B). This correlation agreed with a model where E2f3a promotes expression of group A genes in growing myoblasts and $\mathrm{E} 2 \mathrm{f} 3 \mathrm{~b}$ functions as a transcriptional repressor in myotubes. However, the differentiation-associated repression of group B genes, such as Top2a (Supplemental Fig. S4A), cannot be attributed to E2f3b and must be mediated by some other repressor, possibly E2f4, as the overlap between group B genes and E2f4 targets is extensive.

The E2f3b-specific target genes in groups C-E did not exhibit such a strong correlation between expression and differentiation, but had an almost even distribution of both up- and down-regulated transcripts (Fig. 4C-E). In contrast to groups A and B, these groups were represented by considerably more functionally diverse categories, such as myogenic differentiation, signal transduction, metabolic pathways, and transcription (Fig. 2E), potentially explaining the highly variable target gene expression patterns that we observed. For example, Smyd1, Tnnc2, and Chrng were induced during differentiation, while $I d 3$ and Ccnb1were down-regulated (Supplemental Fig. S4A).

With the exception of the behavior of group A genes, which constituted a small fraction of the total number of E2f3 targets we identified, the correlation between E2f3a and E2F3b binding and gene expression during differentiation was not fully consistent with a model in which E2f3b functions as a strong transcriptional repressor. Together with the apparent E2f3-independent suppression of group $\mathrm{B}$ genes in myotubes, and the diverse expression profiles of groups C-E, our data indicates that regulation of E2f3 target genes is more complex than anticipated, and that E2f3b may not function as a simple binary switch. 
Asp et al.
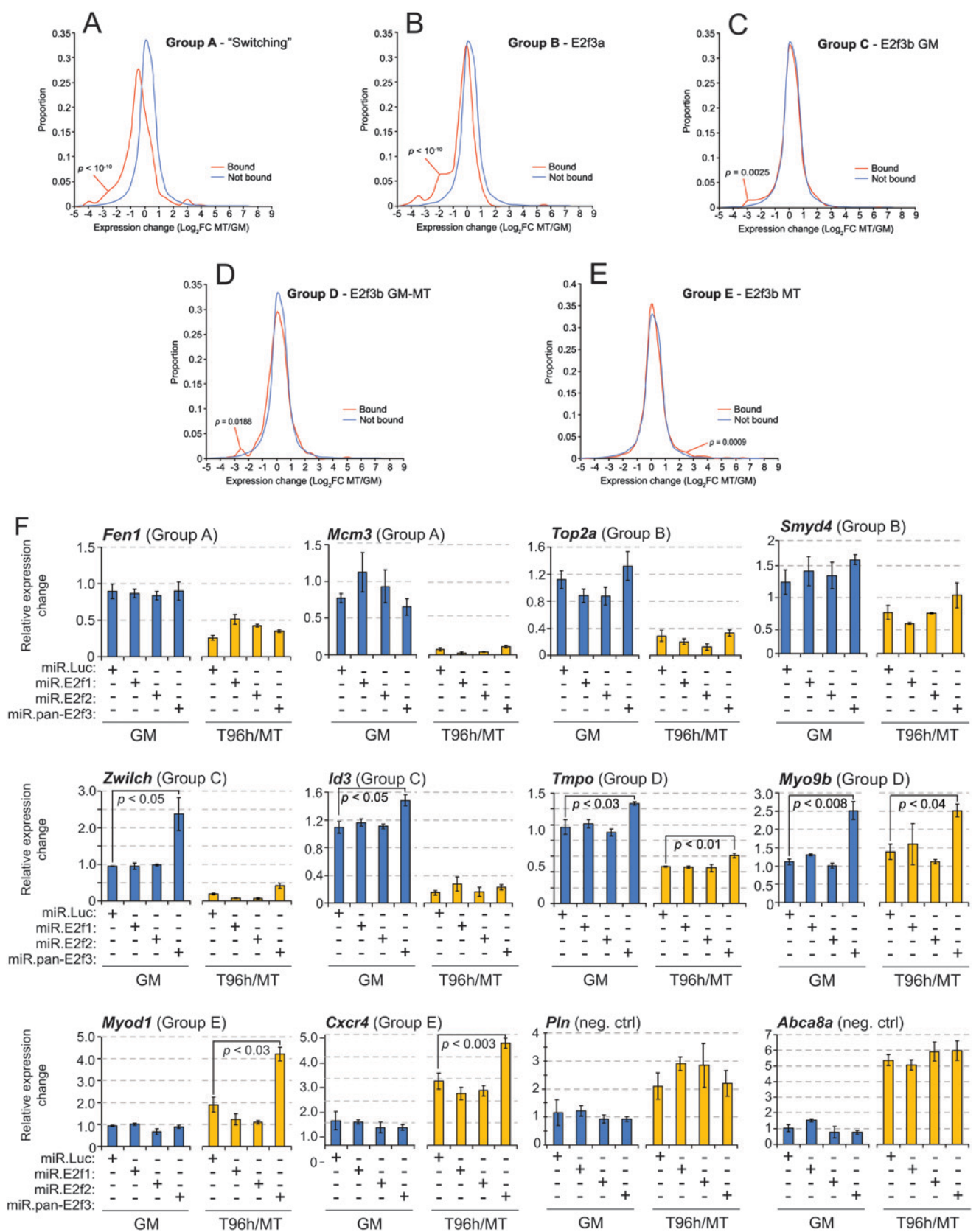

Figure 4. Correlation between E2F3 isoform binding and gene expression during differentiation and changes in E2F3 target gene expression when depleting E2F3. $(A-E)$ Proportion of genes with the indicated fold change in gene expression in myotubes versus growing myoblasts ( $\log _{2} \mathrm{FC} \mathrm{MT/GM)} \mathrm{for} \mathrm{each} \mathrm{group} \mathrm{of} \mathrm{E2F3} \mathrm{targets} \mathrm{compared} \mathrm{with} \mathrm{genes} \mathrm{that} \mathrm{are} \mathrm{bound} \mathrm{(red} \mathrm{trace)} \mathrm{or} \mathrm{not} \mathrm{bound} \mathrm{(blue}$ trace). $P$-values were derived from the hypergeometric distribution test. $(F)$ RT-qPCR analysis of mRNA levels of a select number of E2F3 target genes following E2F3 depletion. Error bars and $P$-values as in Figure 1D.

\section{E2f3 regulates transcription in an isoform-specific manner}

We investigated the impact of ablating E2f3a and E2f3b on the expression of target genes during differentiation by analyzing steady-state mRNA levels in growing myoblasts and myotubes using quantitative real-time PCR (qPCR).

According to a simple binary switching model, group A genes would be expected to respond to E2f3 loss by being down-regulated in growing cells and up-regulated in myotubes, while we would predict group B genes to be down-regulated in myoblasts and unaffected in myotubes. However, for the vast majority of group A and B genes tested, no significant changes were observed upon depleting E2f3, either in myoblasts or myotubes (Fen1, Mcm3, Top2a, and Smyd4) (Fig. 4F). We propose that other activating E2f family member(s) compensate for the loss of E2f3a activation on these genes, as indicated by 
recent studies (Danielian et al. 2008; Tsai et al. 2008). In a similar manner, the differentiation-associated downregulation of these genes in the absence of $\mathrm{E} 2 \mathrm{f} 3 \mathrm{~b}$ could be mediated by E2f4, as the majority of group A and B genes are also targets of this E2F (Fig. 2D).

Interestingly, group $\mathrm{C}$ genes displayed a stronger response to E2f3 depletion than group $\mathrm{A}$ and $\mathrm{B}$ genes, exhibiting significant up-regulation exclusively in myoblasts after knockdown (Fig. 4F). This coincided with the fact that group $\mathrm{C}$ genes were targets of $\mathrm{E} 2 \mathrm{f} 3 \mathrm{~b}$ in myoblasts but not in myotubes. Our data further suggest that E2f3b limits the rate of transcription of these genes, functioning as an attenuator of transcription rather than acting as a strong repressor, as these genes were significantly expressed even in the presence of E2f3b. The correlation between $\mathrm{E} 2 \mathrm{f} 3 \mathrm{~b}$ and transcriptional attenuation was even more apparent for the constitutive E2f3b target genes of group D, which, as a consequence, were significantly upregulated in both myoblasts and myotubes after E2f3 ablation (Tmpo and Myo9b) (Fig. 4F). Interestingly, the myotube-specific E2f3b target genes in group E had the strongest response to E2f3 loss and were significantly upregulated in myotubes. Expression of these genes in myoblasts was unaffected by E2f3 depletion, further strengthening the correlation between E2f3b and attenuation of transcription (Myod1 and Cxcr4) (Fig 4F).

The observed expression changes appear to be specific for E2f3, since depletion of E2f1 or E2f2 did not show a corresponding effect on expression of any of the genes tested in myoblasts or myotubes, in agreement with our observation that these family members are not essential for muscle differentiation (Figs. 1C, 4F). These results also suggest that $\mathrm{E} 2 \mathrm{f} 3 \mathrm{~b}$ is a more critical regulator of transcription than E2f3a during myogenic differentiation, since we observe stronger responses to E2f3b loss in myotubes as compared with E2f3a loss in myoblasts. We propose that other activating E2Fs, such as E2f1 and E2f2, may compensate for the loss of E2f3a on groups A and $\mathrm{B}$ genes in myoblasts, whereas E2f3b plays a role that is not entirely fulfilled by other E2Fs in myotubes. Furthermore, these data reinforce the notion that E2f3b has unique biological properties distinct from those of E2f3a.

\section{$E 2 f 3 b$ is essential for myogenic differentiation}

Our functional analysis of isoform-specific target genes showed that E2f3a was associated with cell cycle and proliferation genes in myoblasts, whereas E2f3b was associated with developmental and differentiation genes in myotubes, particularly those involved in myogenesis. This suggested that $\mathrm{E} 2 \mathrm{f} 3 \mathrm{~b}$ has a more important role in myogenic differentiation than E2f3a. To test this, we assessed the ability of both isoforms to individually rescue the abortive differentiation associated with $\mathrm{E} 2 \mathrm{f} 3$ loss. Attempts to differentially ablate the two isoforms were unsuccessful due to the limited amount of sequence that could be targeted discriminatively (344 bp for E2f3a and $46 \mathrm{bp}$ for E2f3b). Therefore, we took an alternative approach in which we attempted to rescue the differen- tiation phenotype by individually expressing RNAiresistant, HA-tagged E2F3 isoforms in E2f3 miRNAexpressing cells (Materials and Methods). Expression of E2f3a or E2f3b in untreated cells lead to distinctly elevated protein levels, while the levels of E2f3a and E2f3b observed after infecting E2F3 knockdown cells were comparable with those found in untreated myoblasts and myotubes (Supplemental Fig S4B).

First, we gaged the impact of overexpressing either isoform in untreated myoblasts. Unexpectedly, expression of E2f3a had no detectable adverse effects on normal differentiation as compared with untreated and control transduced cells (Supplemental Fig. S5B). This result suggested that there are cellular mechanisms capable of overriding inappropriate E2f3a activity. The same is also true for E2f3b, since its forced expression in untreated cells did not interfere with differentiation (Supplemental Fig. S5B).

Next, we attempted to rescue the differentiation defects observed after E2f3 depletion by expressing E2f3a or E2f3b. Expression of E2f3a in E2f3-depleted cells was not able to rescue the abortive differentiation, but rather, caused a phenotype even more severe than loss of E2f3 alone (cf. Figs. 5A and $1 \mathrm{C}$ ). In striking contrast, expression of E2f3b in E2f3-deficient cells lead to near complete rescue of differentiation and resulted in organized and dense bundles of myotubes (cf. Figs. 5A and 1C). These data strongly suggested that E2f3b, but not E3f3a, was essential for myogenic differentiation, and this conclusion agreed with the functional categories assigned to our E2f3b target genes.

\section{E2f3b promotes myogenic differentiation in a $\mathrm{pRb}$-independent manner}

The role of pocket proteins in myogenic differentiation has been intensively studied both in mouse and in cell culture models, and it is clear that $\mathrm{pRb}$ is essential for myogenic differentiation (Novitch et al. 1996, 1999; Kobayashi et al. 1998; de Bruin et al. 2003; Wu et al. 2003). It has been shown that a population of $\mathrm{E} 2 \mathrm{f} 3 \mathrm{~b}$ specifically interacts with $\mathrm{pRb}$ in quiescent and postmitotic cells, and we showed that this is also observed in myotubes (Supplemental Fig. S5A; Kong et al. 2007). It has also been shown that $\mathrm{pRb}$ is associated with long-term repression of transcription in myotubes (Blais et al. 2007). However, the fact that E2f3b target genes were not exclusively down-regulated during differentiation suggested that E2f3b function in the context of muscle differentiation could be at least partially $\mathrm{pRb}$ independent.

To test this hypothesis, we introduced a point mutation into our miRNA-resistant E2f3a and E2f3b expression constructs ( $\mathrm{Y} 436 \mathrm{H}$ in E2f3a and $\mathrm{Y} 309 \mathrm{H}$ in E2f3b) (Materials and Methods). The targeted residue is conserved among E2F1-3, and this substitution has been shown to disrupt the interaction between $\mathrm{E} 2 \mathrm{fl}$ and $\mathrm{pRb}$ while preserving trans-activation function (Shan et al. 1996). Expression of these miRNA-resistant HA-tagged E2f3 mutants resulted in levels that approximated both their wild-type counterparts and the endogenous proteins 

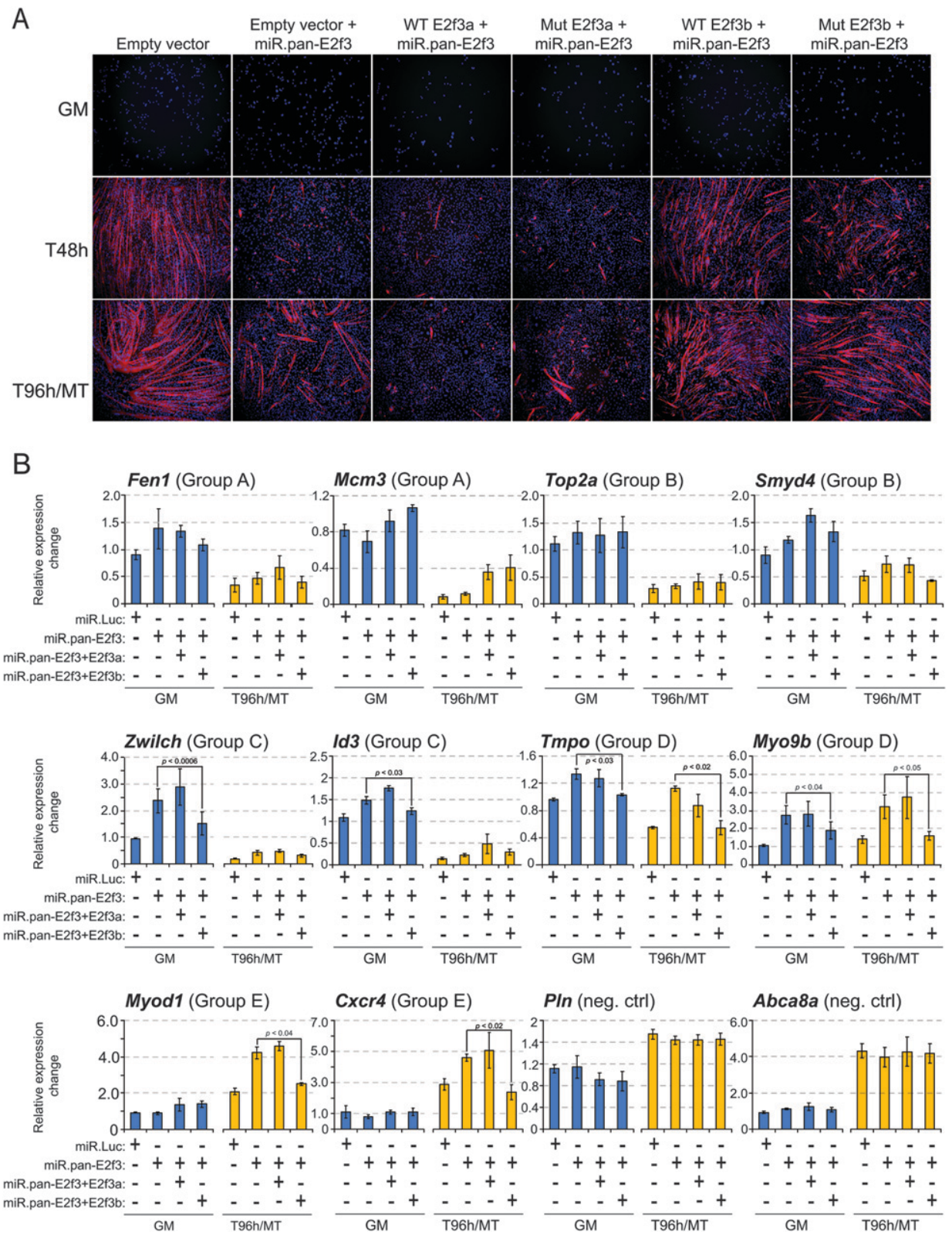

Figure 5. E2f3b is uniquely required to promote myogenic differentiation and restores normal expression of target genes. $(A)$ Differentiation was scored by staining for MHC and nuclei were visualized with DAPI as described in Figure 1. Combinations of virus transductions are indicated on the top. (B) RT-qPCR analysis of the genes in Figure 4 after forced expression of either E2f3a or E2f3b in E2F3-depleted cells. Error bars and $P$-values as in Figure 1D.

(Supplemental Fig. S4B). We verified that their interactions with endogenous $\mathrm{pRb}$ were disrupted by immunoprecipitating both wild-type and mutant E2f3 proteins via the HA tag and analyzing coprecipitated pRb by Western blotting. Wild-type E2f3a and E2f3b clearly coprecipitated endogenous $\mathrm{pRb}$, while the corresponding mutants did not (Supplemental Fig. S4C).

Next, we attempted to rescue the differentiation defect provoked by E2f3 loss by expressing the E2f3a and E2f3b pRb-binding-deficient mutants in E2f3 miRNA-expressing cells. Surprisingly, overexpression of mutant E2f3a in untreated cells did not impair differentiation (Supplemen- tal Fig. S5B), despite the fact that pRb-uncoupled, constitutive E2f3a activity was expected to adversely affect cell cycle exit and inhibit differentiation. Furthermore, overexpression of mutant E2f3b did not have any detectable effect on differentiation (Supplemental Fig. S5B).

Expression of mutant E2f3a in E2f3-deficient cells failed to restore differentiation but instead caused a phenotype even more severe than the wild-type protein (Fig. 5A). In sharp contrast, an E2f3b mutant unable to bind $\mathrm{pRb}$ rescued differentiation as well as the wild-type protein (Fig. 5A), demonstrating that $\mathrm{E} 2 \mathrm{f} 3 \mathrm{~b}$ function in myogenic differentiation is independent of $\mathrm{pRb}$. 


\section{E2f3b restores expression of target genes}

We investigated the expression of E2f3 target genes in the isoform-specific rescue experiments described above by analyzing steady-state mRNA levels using qPCR before and after rescue. Forced expression of E2f3a in E2f3depleted cells did not lead to dramatic changes in expression levels of any E2f3 target genes investigated, neither in myoblasts nor myotubes, and the weak changes detected were not statistically significant (Fig. 5B). However, the ability of E2f3b to rescue differentiation coincided with significant transcriptional down-regulation and restoration of normal transcript levels of $\mathrm{E} 2 \mathrm{f} 3 \mathrm{~b}$ targets (Fig. 5B). This effect was most prominent for E2f3b target genes in myotubes (Myod1 and Cxcr4) (Fig. $5 \mathrm{~B})$ suggesting a context-dependent role for $\mathrm{E} 2 \mathrm{f} 3 \mathrm{~b}$ in agreement with its ability to promote differentiation. In sharp contrast, expression of group A and B genes did not change significantly upon restoration of E2f3b (Fen 1, Mcm3, Top2a, and Smyd4) (Fig. 5B), which was also the case when total E2f3 was depleted (Fig. 4F).

Although E2f3a is expressed at slightly higher levels than E2f3b in the rescue experiment (Supplemental Fig. S4), our data showed remarkably specific regulation by E2f3 isoforms, since even these higher levels of E2f3a were unable to significantly affect transcription of the E2f3 target genes analyzed. In contrast, the lower levels of ectopically produced E2f3b were clearly able to attenuate expression of its targets, restoring their expression to normal levels.

\section{Gene-specific variations in E2f3 isoform targeting}

We performed conventional E2f3 ChIP analysis on the rescue experiments described above to correlate the observed restoration of normal expression with E2f3 isoform-specific promoter binding.

Exogenous E2f3a was efficiently recruited to groups A and $\mathrm{B}$ genes in myoblasts, even though it did not induce statistically significant changes in expression (Fen1, Mcm3, Top2a, and Smyd4 in Fig. 6). This agrees with the proposed redundancy between E2f-1, E2f-2, and E2f-3a on these genes, ensuring full expression even in the absence of one of these activators. We also detected exogenous E2f3a binding upon ectopic expression of this isoform in myotubes, wherein there is normally no E2f3a protein present. However, this nonphysiological binding was not functional, since it did not result in altered gene expression. Moreover, even though we could only detect significant recruitment of E2 $\mathrm{f} 3 \mathrm{~b}$ to two group A and B genes out of the four analyzed (Fen and Smyd4) (Fig. 6) binding was only observed in myotubes but not in myoblasts, although the protein was expressed at levels equivalent to those of E2f3a. However, E2f3b binding was not crucial for the proper expression of these genes as they were all down-regulated during differentiation in both E2f3-depleted and E2f3b-rescued cells. This supports our conclusion that there is functional overlap between $\mathrm{E} 2 \mathrm{f} 3 \mathrm{~b}$ and E2f4 on these genes and that E2f4 alone is enough to ensure differentiation-associated transcriptional repression and cell cycle exit.
For genes in groups $\mathrm{C}$ and $\mathrm{D}$ we detected significant recruitment of both $\mathrm{E} 2 \mathrm{f} 3 \mathrm{a}$ and $\mathrm{E} 2 \mathrm{f} 3 \mathrm{~b}$ in the rescued cells. As for group A and B genes, binding of E2f3a occurred in both myoblasts and myotubes, but no significant changes in expression were associated with this binding (Fig. 6). For group C genes, E2f3b binding was only detected in myoblasts and coincided with the time when endogenous E2f3b would normally be recruited to these genes (Zwilch, Id3 in Fig. 6) and similarly, E2f3b recruitment to group $\mathrm{D}$ genes mirrored the pattern of the endogenous protein and was detected in both myoblasts and myotubes (Tmpo, Myo9b in Fig. 6). For both group $C$ and group $D$ genes, recruitment of $E 2 f 3 b$ restored expression to normal levels compared with E2f3 depleted cells.

Strikingly, group E genes showed a very high degree of specificity for $E 2 \mathrm{f} 3 \mathrm{~b}$, and in no case did we detect binding of E2f3a to these genes. Recruitment of E2f3b mirrored precisely the normal pattern of $\mathrm{E} 2 \mathrm{f} 3 \mathrm{~b}$ recruitment, since we exclusively observed strong binding in myotubes (Myod1 and Cxcr4 in Fig. 6). As shown in Figure 5B, E2f3b binding to these genes was accompanied by complete restoration of normal expression levels.

Thus, E2f3b exhibited remarkably specific and physiological patterns of promoter recruitment and regulation of its targets. Likewise, E2f3a recruitment to promoters was enabled by re-expression of this isoform, except on group E genes. In all cases, however, inappropriate gene regulation was not observed, attesting to additional mechanisms that limit or prevent inappropriate activity of E2f3a on these genes. The mechanisms that restrict E2f3a and E2f3b binding and activity are currently unknown, but the fact that we only detect transcriptional changes associated with E2f3b binding on E2f3b-specific genes supports our conclusion that $\mathrm{E} 2 \mathrm{f} 3 \mathrm{~b}$ is both functionally unique and essential for myogenesis.

\section{Discussion}

Using methods that enhance the ability to identify family member-specific functions (isoform-specific ChIP-onchip and acute ablation), our work highlights both specificity and redundancy within the E2F family. Here, we defined a previously unknown and critical role for E2f3b in myogenic differentiation. Identification of E2f3 isoform-specific target genes uncovered a hitherto unknown complexity in E2F3 function that vastly extends the network of gene categories controlled by the E2F transcription factor family.

\section{E2F factors in myogenic differentiation}

Our data show that E2f3b plays an indispensable role in myogenic differentiation, while, in striking contrast, E2f1, E2f2, and E2f3a do not play an obvious role in our in vitro differentiation model. Genes targeted by E2f3a are heavily enriched in proliferation-associated genes, while E2f3b targets are heavily enriched in genes involved in differentiation, signaling, lipid biosynthesis, and cell adhesion and motility, suggesting that E2f3b is uniquely 

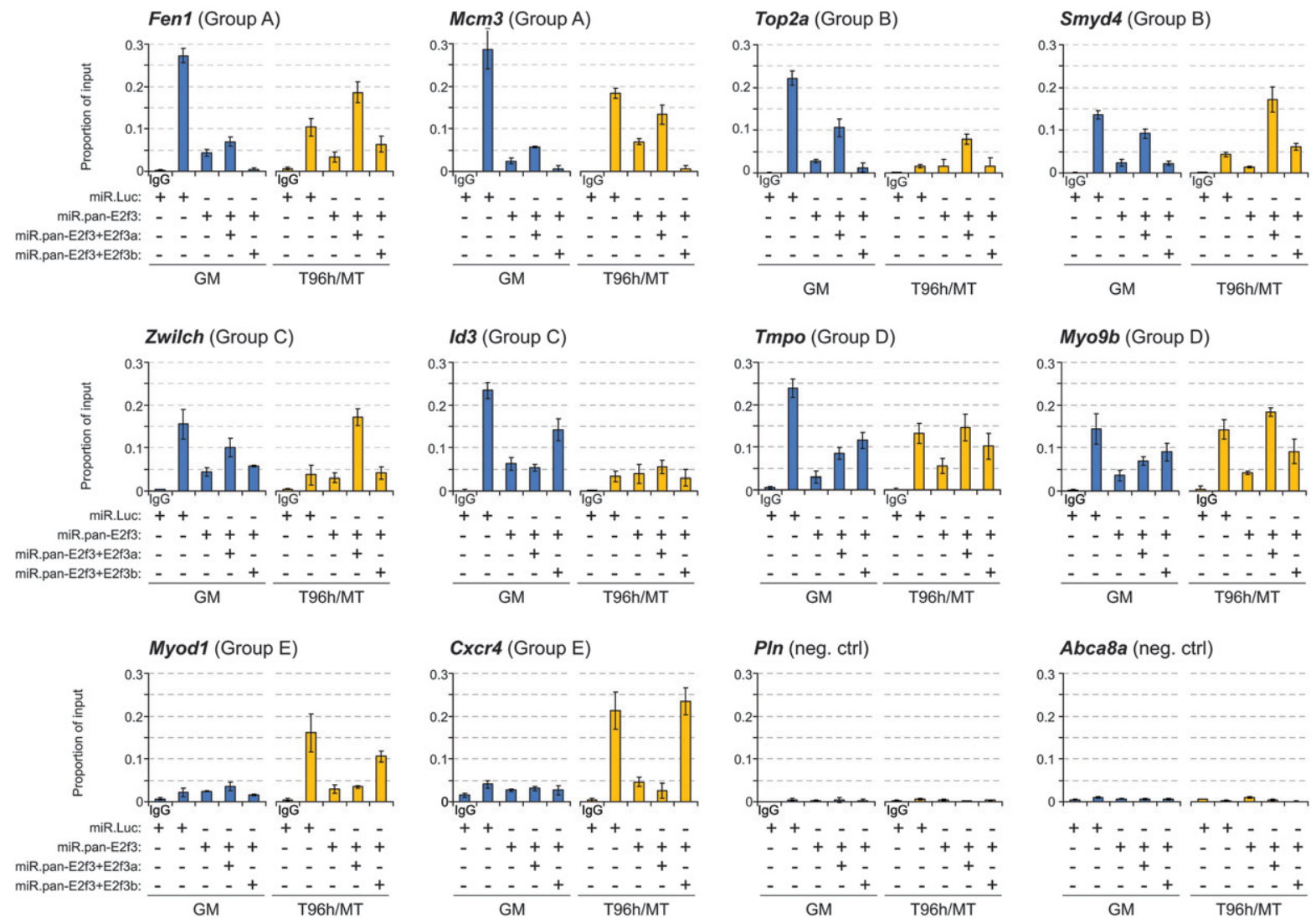

Figure 6. ChIP analysis of promoter binding by E2f3 isoforms in the rescue experiment in Figure 5. Chromatin was prepared from each combination of transduced $\mathrm{C} 2 \mathrm{C} 12$ cells as described and ChIP was performed using the polyclonal E2f3 antibody that recognizes both isoforms in order to ensure equal enrichment for E2f3a and E2f3b. Enriched DNA was analyzed by qPCR, and error bars depict the standard error of the mean (SEM) derived from three independent experiments.

required for myogenic differentiation (Fig. 7A). In agreement with these observations, our isoform-specific rescue experiment clearly demonstrates that E2f3b is indeed essential for myogenic differentiation.

Although we demonstrated an essential role for E2f3b in myogenic differentiation, we cannot rule out a role for E2f4, since its depletion leads to a mildly hypertrophic phenotype. However, unlike E2f3b, E2f4 does not appear to play a major or nonoverlapping role in differentiation and, at least in the short term, it cannot compensate for E2f3 following acute depletion of this protein. Instead, we believe that E2f4 plays a role in initiating and maintaining the differentiated state, in part by permanently silencing expression of cell cycle genes (Blais et al. 2007; van Oevelen et al. 2008), many of which are bound by the activator E2f3a in myoblasts (A and $\mathrm{B}$ groups).

E2F activity is regulated by pocket proteins such as the retinoblastoma protein. $\mathrm{pRb}$ has a well-established and critical role in myogenic differentiation, and $\mathrm{Rb}$-null mice have severe defects in both skeletal and cardiac muscle development (Wu et al. 2001; Cloud et al. 2002; de Bruin et al. 2003). The $\mathrm{pRb}$ protein is critical for initiating and maintaining myogenic differentiation (Novitch et al. 1996, 1999; Kobayashi et al. 1998; Blais et al. 2007). However, our work suggests that E2f3b can efficiently promote myogenic differentiation in a pRb-independent manner. Thus, our studies suggest an additional layer of regulation whereby E2f3b promotes events outside the cell cycle (i.e., myogenic differentiation) in a pRb-independent manner.

\section{Selectivity and redundancy among the E2F activator class}

Several lines of evidence suggest that our genome-wide and phenotypic studies have identified bona fide E2f3 isoform-specific targets in myotubes. First, we found that E2f3a essentially disappears during myogenic differentiation and in accordance with this observation, our ChIPon-chip studies identified very few E2f3a targets in myotubes. None of these putative targets could be verified by confirmatory ChIP, indicating that they were false positives (data not shown). In addition, genes that otherwise are strong targets of E2f3a in myoblasts are not bound by the residual E2f3a protein present in myotubes, indicating that E2f3a binding to target genes is limited to myoblasts. Myoblast-specific E2f3a target genes (groups $A$ and B) are involved in cell growth and proliferation and are subsequently strongly suppressed in myotubes, which coincides with binding of E2f3b to most of these genes. 
A

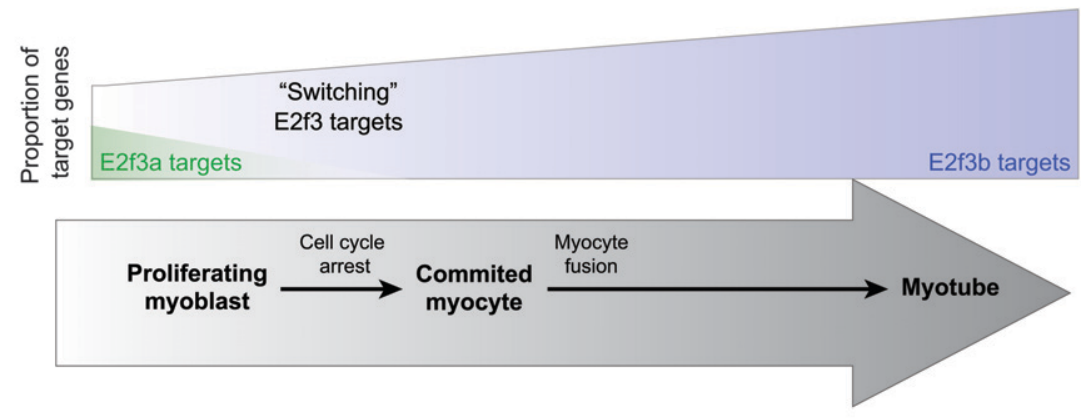

B

MYOBLASTS
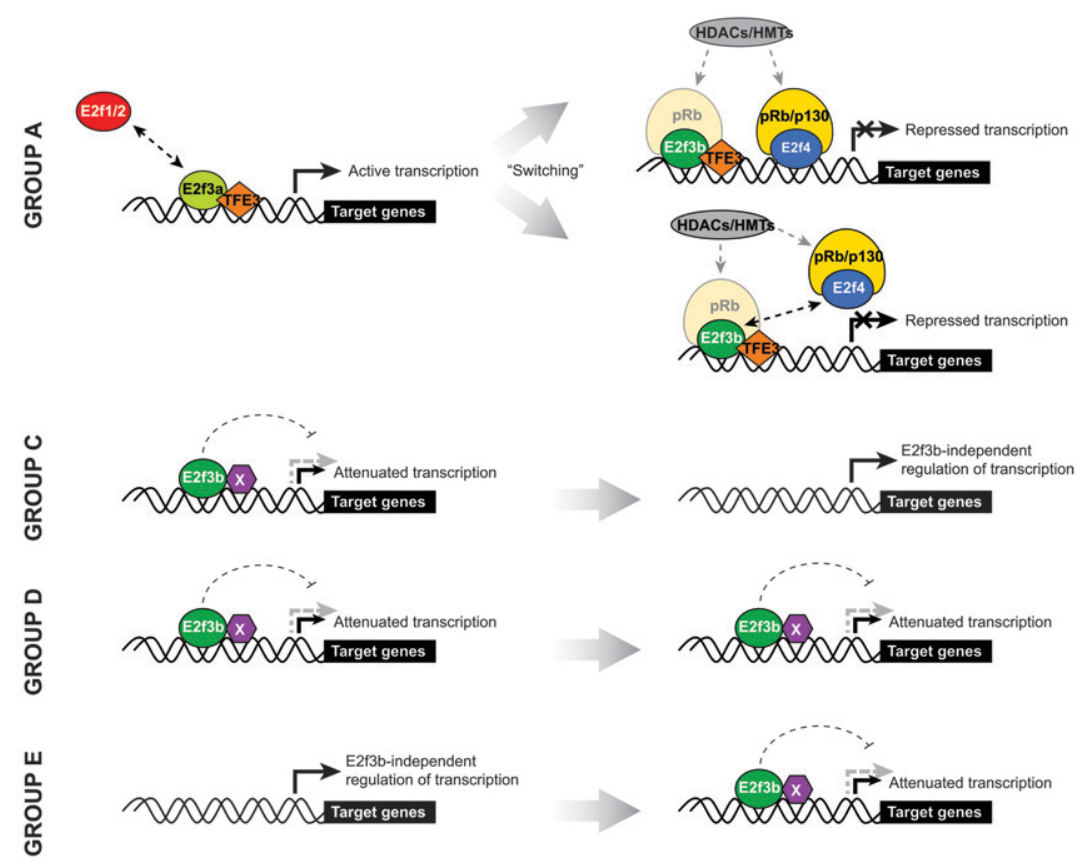

Figure 7. Model describing the role of E2F and pocket protein family members in myogenic differentiation. (A) Relative changes in the amount of E2f3a and E2f3b target genes during differentiation. $(B)$ Model differential E2F3 isoform binding during myogenesis depicting the proposed existence of an E2f3b-specific targeting partner (X, purple hexagon).
However, the majority of these genes are also targeted by E2f4 in myotubes, and repression is most likely mediated through the redundant action of E2f4 and E2f3b, since depletion of $\mathrm{E} 2 \mathrm{f} 3 \mathrm{~b}$ does not cause significant derepression of these genes nor failure to arrest the cell cycle (Figs. 1, 4B). Second, "subtraction" of targets bound by E2f3a from the list of $\mathrm{E} 2 \mathrm{f} 3 \mathrm{a} / \mathrm{b}$ targets resulted in a cadre of genes ("E2f3b targets") whose functional annotations are remarkably distinct from those ascribed to E2f3a. Indeed, the list of functional annotations indicates that $\mathrm{E} 2 \mathrm{f} 3 \mathrm{~b}$ plays a preferential role in differentiation, while E2f3a exerts control over cell cycle-related events. Third, E2f3a and $\mathrm{E} 2 \mathrm{f} 3 \mathrm{~b}$ targets are differentially regulated during myogenesis (Fig. 4A).

Moreover, our studies revealed redundancies in E2f3amediated activation, since acute knockdown of E2F3 does not significantly affect expression of genes specifically bound and activated by E2f3a in myoblasts. This suggests that other factors, including E2F1 and E2F2, may be able to compensate for the absence of E2f3a. This was further suggested by the absence of loss of expression when depleting E2F1 or E2F2, supporting a model where these three activating E2Fs are multiredundant and normally function in concert to ensure correct gene expression.

In contrast, we observed far less prominent compensation for E2f3b activity by other E2Fs, as E2f3bspecific target genes were significantly up-regulated upon loss of E2f3, and proper gene regulation was restored solely upon re-expression of $\mathrm{E} 2 \mathrm{f} 3 \mathrm{~b}$ in a condition-specific manner. Importantly, E2f3b, but not E2f3a expression was uniquely able to promote myogenic differentiation. While our experiments were in progress, another study examined the extent to which members of the E2F activator class compensate for one another by ablating all three E2F activators (E2F1-3) in mice and by expressing $E 2 f 1$ or $E 2 f 3 b$ from the $E 2 f 3 a$ locus (Tsai et al. 2008). In this study, a single activator protein, 
E2f3a, was sufficient to ensure embryonic and postnatal development. Further, these knock-in experiments suggested that E2F family members are interchangeable, since expression of either E2f1 or E2f3b rescued phenotypes associated with $E 2 f 3 a$ inactivation, but timing of expression dictated by $E 2 f 3 a$ regulatory sequences was paramount. We showed that in the context of muscle differentiation, E2f3b generally functions as an attenuator of transcription and not as an activator, which is difficult to reconcile with the presumptive ability of E2f3b to rescue the E2f3a phenotype described by Tsai et al. (2008). However, we did not examine all E2f3b target genes, and therefore we cannot exclude the possibility that there might be instances when $\mathrm{E} 2 \mathrm{f} 3 \mathrm{~b}$ functions as an activator of critical developmental genes.

No muscle defects were described in the knock-out/ knock-in study by Tsai et al. (2008) nor was a muscle phenotype detected in a recent publication describing E2F3 isoform knockout mice in an otherwise wild-type E2F background (Danielian et al. 2008). We believe that a likely explanation pertains to the manner in which E2f3 was ablated. In our case, ablation was acute, whereas the earlier study took advantage of germline knockouts, a situation in which significant compensation by other E2F factors is known to occur (Kong et al. 2007). In preliminary studies, we tested this possibility by continuously cultivating myoblasts depleted for E2F3 for 2-3 wk before inducing differentiation. Interestingly, silencing of E2F3 expression persists for the entire duration of this experiment, but after such extended culture, these myoblasts partially regain their ability to differentiate, albeit not as well as wild-type cells (data not shown). This provides evidence for the differences between acute and long-term loss of E2F factors and suggests that care must be taken when analyzing such data.

In the mouse knockout/knock-in models, it is conceivable that most cells are able to compensate for lack of E2f3a, and thereby enable the animal to complete embryogenesis. However, it is clear that this is not the case for all tissues, since $E 2 f 3 a^{-1-}$ mice are deficient in white adipose tissue. Intriguingly, we find that both E2F3 isoforms are recruited to a substantial number of genes involved in lipid metabolism (Fig. 2E), prompting future experiments designed to investigate whether deregulation of these target genes could, in part, account for the mutant phenotype.

\section{Factors underlying E2f3a and E2f3b specificity}

It has been shown that E2f3a synergistically interacts with the E-box transcription factor TFE3 and that this interaction promotes specific recruitment of E2F3 to the p68 promoter and activates transcription /Giangrande et al. 2003). This activation can be negated by coexpression of E2f3b, which agrees with our experiments that show that E2f3b is able to attenuate gene expression, most prominently in myotubes (Figs. 5B, 7). Given this result, it is interesting to speculate that a specificity factor akin to TFE 3 could also operate in recruiting E2f3b to target genes
(Fig. 7B). Isoform specificity could then be imparted by the disappearance of E2f3a (and presence of E2f3b) as cells differentiate. Such a mechanism has been observed for the Arf promoter, which is normally specifically bound by $\mathrm{E} 2 \mathrm{f} 3 \mathrm{~b}$, but becomes occupied by E2f3a in the absence of E2f3b (Danielian et al. 2008). However, this model does not explain the existence of E2f3b-specific target genes in myoblasts, wherein both isoforms are present, which suggests that $\mathrm{E} 2 \mathrm{f} 3 \mathrm{~b}$ can be uniquely targeted to promoters as suggested in our model (Fig. 7B).

The combined analysis of isoform recruitment and expression in our rescue experiment clearly demonstrates both specific and nonspecific recruitment and function of isoforms to E2f3 target genes. E2f3a is far more promiscuous than E2f3b and is able to bind to most genes in both myoblasts and myotubes, with the exception of group E genes that appear to be resistant to E2f3a binding. On the other hand, E2f3b is highly specific in its binding to chromatin, and the ectopically expressed protein precisely mirrored the binding normally seen with endogenous E2f3b. Interestingly, despite being able to bind to most genes investigated, E2f3a had no detectable effect on the transcription of these genes. For groups $\mathrm{A}$ and $\mathrm{B}$ genes, this can be explained by the proposed functional redundancy with E2f1 and E2f2, which would ensure that these genes are always transcribed at optimal levels irrespective of E2f3a. However, such a model does not explain why E2f3a binding to groups $\mathrm{A}$ and $\mathrm{B}$ genes in myotubes has no affect on transcription or why groups $\mathrm{C}$ and $\mathrm{D}$ genes are similarly resistant to transcriptional activation by E2f3a. Currently, we do not know the mechanism behind this selectivity. For $\mathrm{E} 2 \mathrm{f} 3 \mathrm{~b}$, the situation is strikingly different, and binding by the exogenous protein restores normal expression levels in all cases investigated, except for group A genes in myotubes, where E2f3b binding appears to have no effect on transcription. We believe that the proposed redundancy with E2f4 allows for the differentiationassociated repression of these genes in the presence or absence of E2f3b. The discovery that group E genes are specific for $\mathrm{E} 2 \mathrm{f} 3 \mathrm{~b}$, with respect to both binding and gene regulation, strongly supports our conclusion that E2f3b is essential for myogenic differentiation, since this group contains myotube-specific E2f3b targets involved in differentiation.

The work reported here documents that regulation by E2f3a and E2f3b is remarkably robust and highly specific. We propose that function and recruitment of a specific isoform depends on the individual properties of E2F3 target promoters. Indeed, preliminary analyses have revealed striking differences between groups A and B promoters on the one hand and groups $\mathrm{C}-\mathrm{E}$ promoters on the other. For instance, while consensus E2f-binding sites are abundant in the former group, recognizable E2f-binding sites are essentially absent in the latter (data not shown). We are currently performing detailed computational and functional analyses on each group of target promoters in an attempt to identify isoform-specific binding partners and to determine the mechanism by which E2f3a and E2f3b specifically regulate transcription (Fig. 7B). 


\section{Materials and methods}

\section{Tissue culture}

C2C12 myoblasts were obtained from Sigma and cell culture, differentiation, and selective harvesting of myotubes were as described previously (Blais et al. 2007).

\section{Plasmids}

Standard molecular biology methods were used for all DNA manipulations and all constructs were verified by sequencing and extensive restriction digestions. For RNAi we used a modified version of the miRNA expression system available through Open Biosystems (Dickins et al. 2005; Silva et al. 2005). We modified the pMSCV-TMP vector by replacing the Tet-responsive promoter with a constitutive mouse $\mathrm{E} 1 \alpha$ promoter. miRNA sequences targeting different E2Fs were obtained through the RNAi Codex database (http://codex.cshl.edu/scripts/newmain. pl) and target specificity was verified by BLAST searches of the mouse genome. The 97-mer miRNA sequences given by the database were obtained from Operon and cloned into the miRNA vector according to the instructions from Open Biosystems. The ORFs of E2f3a and E2f3b were PCR-amplified from C2C12 myoblast cDNA and cloned into pBABE-puro. We created miRNAresistant expression vectors by modifying the miRNA targeting sequence by introducing three silent base changes by PCR. These constructs were further modified by PCR to generate point mutations that disrupted the interaction with $\mathrm{pRb}$ as described previously (Shan et al. 1996). Primer and miRNA-oligo sequences are available upon request.

\section{Viral infections}

Retroviral particles were produced in the packaging cell line Phoenix-Eco as described previously (Acosta-Alvear et al. 2007). C2C12 cells were transduced with $3 \times 1 \mathrm{~mL}$ of viral supernatant every $4 \mathrm{~h}$ supplemented with $8 \mu \mathrm{g} / \mathrm{mL}$ polybrene. The last infection was left overnight and replaced with fresh medium the next morning, and cells were allowed to recover for $10 \mathrm{~h}$, after which puromycin was added at a concentration of $2 \mu \mathrm{g} / \mathrm{mL}$. Cells were selected for $3 \mathrm{~d}$ and split as needed to keep them as growing myoblasts, after which they were divided into aliquots and stored in liquid nitrogen. For double infections, the total volume of virus supernatant was kept constant at $1 \mathrm{~mL}$, and cells were transduced simultaneously with two different viruses.

\section{FACS analysis}

FACS profiling was performed as described previously (Tsang et al. 2007).

\section{ChIP and ChIP-on-chip}

ChIP and ChIP-on-chip were performed as described previously (Blais et al. 2005; Acosta-Alvear et al. 2007). Conventional ChIP analyses were performed three to four times and ChIP-on-chip experiments were performed in duplicates, in all cases using chromatin samples from independent experiments. Microarrays were scanned using an Agilent DNA Microarray Scanner (model G2565Ba). The data was processed using the Agilent feature extraction software (version 9.5.2) and Agilent ChIP analytics version 1.31. Data were analyzed using a heuristic peak-finding algorithm developed in our laboratory for promoter and tiling arrays, and highly stringent cut-offs were used to determine significant binding (Supplemental Fig. S1D).

\section{Immunoprecipitation}

Nuclear extracts were prepared as described previously (Cavellan et al. 2006) with the following modifications: Hypotonic homogenization buffer was adjusted to $7 \mathrm{mM} \mathrm{KCl}$ and nuclear extraction buffer was adjusted to $0.42 \mathrm{M} \mathrm{KCl}$. A total of $1 \mathrm{mg}$ of nuclear extract was immunoprecipitated with $1 \mu \mathrm{g}$ of antibodies cross-linked to a mix of protein $\mathrm{A} / \mathrm{G}$ sepharose and washed with nuclear extraction buffer containing $0.15 \mathrm{M} \mathrm{KCl}$. Precipitated material was separated on an $8 \%$ SDS-PAGE together with $50 \mu \mathrm{g}$ of nuclear extract as input and blotted to PVDF.

\section{$R T-P C R$}

RNA isolation and reverse transcription were performed as described previously (Acosta-Alvear et al. 2007).

\section{Real-time PCR}

Conventional ChIP and RT-PCR analysis were performed by qPCR using SYBR green and the Bio-Rad iCycler (version 4.0006). All primer pairs were tested using dilution series of template (chromatin or cDNA) to verify specificity and determine their amplification efficiency ( $E$-value). $E$-values were used to calculate amplification fold changes in accordance with the Pfaffl method (Pfaffl 2001). Conventional ChIP enrichment was calculated as the relative amplification fold change to respective input (myoblasts or myotubes) and expressed as a proportion of the input amplification. Inputs represent $0.25 \%$ of the total chromatin in the ChIP $(25 \mu \mathrm{g})$. An enrichment of $0.05 \%$ or higher was empirically determined to represent significant binding. Expression data were calculated relative to untreated myoblasts, normalized against Rps26, and expressed as proportion fold change relative to untreated myoblasts. Primer sequences for ChIP and RT-PCR are available upon request.

\section{Immunofluorescence microscopy and BrdU labeling}

Immunofluorescence staining of MHC and BrdU labeling and detection were performed as described (Blais et al. 2007), except that BrdU labeling time was shortened to $1 \mathrm{~h}$.

\section{Antibodies}

Antibodies for ChIP and ChIP-on-chip: $\alpha$ E2f3a (MS-1063, Neomarkers) and E2f3a/b (sc-878, Santa Cruz Biotechnologies). Antibodies for Western blot: $\alpha$ E2F1 (sc-193, Santa Cruz Biotechnologies), $\alpha$ E2F2 (sc-633, Santa Cruz Biotechnologies), $\alpha$ E2F4 (sc-512, Santa Cruz Biotechnologies), $\alpha$ GFP (G1546, Sigma-Aldrich) $\alpha$ - $\beta$ Actin (A1978, Sigma-Aldrich) and $\alpha-\alpha$-tubulin (T5168, Sigma-Aldrich). Antibodies for protein immunoprecipitation: $\alpha \mathrm{HA}$ (12CA5). Antibodies for immunofluorescence: $\alpha$ MHC (MF20, Developmental Studies Hybridoma Bank [DSHB]) and $\alpha \mathrm{BrdU}$ (G3G4, DSHB).

\section{Acknowledgments}

We thank current and previous members of the Dynlacht laboratory for support, insightful questions, and comments. This work was supported by grants from the NIH to B.D.D, an American Heart Association Heritage post-doctoral fellowship awarded to P.A., and a Susan G. Komen post-doctoral fellowship awarded to C.v.O. 


\section{References}

Acosta-Alvear, D., Zhou, Y., Blais, A., Tsikitis, M., Lents, N.H., Arias, C., Lennon, C.J., Kluger, Y., and Dynlacht, B.D. 2007. $\mathrm{XBP} 1$ controls diverse cell type- and condition-specific transcriptional regulatory networks. Mol. Cell 27: 53-66.

Aslanian, A., Iaquinta, P.J., Verona, R., and Lees, J.A. 2004. Repression of the Arf tumor suppressor by E2F3 is required for normal cell cycle kinetics. Genes \& Dev. 18: 1413-1422.

Blais, A. and Dynlacht, B.D. 2007. E2F-associated chromatin modifiers and cell cycle control. Curr. Opin. Cell Biol. 19: 658-662.

Blais, A., Tsikitis, M., Acosta-Alvear, D., Sharan, R., Kluger, Y., and Dynlacht, B.D. 2005. An initial blueprint for myogenic differentiation. Genes \& Dev. 19: 553-569.

Blais, A., van Oevelen, C.J., Margueron, R., Acosta-Alvear, D., and Dynlacht, B.D. 2007. Retinoblastoma tumor suppressor protein-dependent methylation of histone $\mathrm{H} 3$ lysine 27 is associated with irreversible cell cycle exit. J. Cell Biol. 179: 1399-1412.

Cam, H., and Dynlacht, B.D. 2003. Emerging roles for E2F: Beyond the G1/S transition and DNA replication. Cancer Cell 3: 311-316.

Cavellan, E., Asp, P., Percipalle, P., and Farrants, A.K. 2006. The WSTF-SNF2h chromatin remodeling complex interacts with several nuclear proteins in transcription. J. Biol. Chem. 281: 16264-16271.

Chen, D., Opavsky, R., Pacal, M., Tanimoto, N., Wenzel, P., Seeliger, M.W., Leone, G., and Bremner, R. 2007. Rb-mediated neuronal differentiation through cell-cycle-independent regulation of E2f3a. PLoS Biol. 5: e179, doi:10.1371/journal.pbio.0050179.

Cloud, J.E., Rogers, C., Reza, T.L., Ziebold, U., Stone, J.R., Picard, M.H., Caron, A.M., Bronson, R.T., and Lees, J.A. 2002. Mutant mouse models reveal the relative roles of E2F1 and E2F3 in vivo. Mol. Cell. Biol. 22: 2663-2672.

Danielian, P.S., Friesenhahn, L.B., Faust, A.M., West, J.C., Caron, A.M., Bronson, R.T., and Lees, J.A. 2008. E2f3a and E2f3b make overlapping but different contributions to total E2f3 activity. Oncogene 27: 6561-6570.

de Bruin, A., Wu, L., Saavedra, H.I., Wilson, P., Yang, Y., Rosol, T.J., Weinstein, M., Robinson, M.L., and Leone, G. 2003. Rb function in extraembryonic lineages suppresses apoptosis in the CNS of Rb-deficient mice. Proc. Natl. Acad. Sci. 100: 6546-6551.

De Falco, G., Comes, F., and Simone, C. 2006. pRb: Master of differentiation. Coupling irreversible cell cycle withdrawal with induction of muscle-specific transcription. Oncogene 25: 5244-5249.

Dickins, R.A., Hemann, M.T., Zilfou, J.T., Simpson, D.R., Ibarra, I., Hannon, G.J., and Lowe, S.W. 2005. Probing tumor phenotypes using stable and regulated synthetic microRNA precursors. Nat. Genet. 37: 1289-1295.

Dimova, D.K., Stevaux, O., Frolov, M.V., and Dyson, N.J. 2003. Cell cycle-dependent and cell cycle-independent control of transcription by the Drosophila E2F/RB pathway. Genes \& Dev. 17: 2308-2320.

Dirlam, A., Spike, B.T., and Macleod, K.F. 2007. Deregulated E2f2 underlies cell cycle and maturation defects in retinoblastoma null erythroblasts. Mol. Cell. Biol. 27: 8713-8728.

Giacinti, C. and Giordano, A. 2006. RB and cell cycle progression. Oncogene 25: 5220-5227.

Giangrande, P.H., Hallstrom, T.C., Tunyaplin, C., Calame, K., and Nevins, J.R. 2003. Identification of E-box factor TFE3 as a functional partner for the E2F3 transcription factor. Mol. Cell. Biol. 23: 3707-3720.
He, Y., Armanious, M.K., Thomas, M.J., and Cress, W.D. 2000. Identification of E2F-3B, an alternative form of E2F-3 lacking a conserved N-terminal region. Oncogene 19: 3422-3433.

Kobayashi, M., Yamauchi, Y., and Tanaka, A. 1998. Stable expression of antisense Rb-1 RNA inhibits terminal differentiation of mouse myoblast C2 cells. Exp. Cell Res. 239: 40-49.

Kong, L.J., Chang, J.T., Bild, A.H., and Nevins, J.R. 2007. Compensation and specificity of function within the E2F family. Oncogene 26: 321-327.

Lee, T.I., Jenner, R.G., Boyer, L.A., Guenther, M.G., Levine, S.S., Kumar, R.M., Chevalier, B., Johnstone, S.E., Cole, M.F., Isono, K., et al. 2006. Control of developmental regulators by Polycomb in human embryonic stem cells. Cell 125: 301313.

Leone, G., Nuckolls, F., Ishida, S., Adams, M., Sears, R., Jakoi, L., Miron, A., and Nevins, J.R. 2000. Identification of a novel E2F3 product suggests a mechanism for determining specificity of repression by Rb proteins. Mol. Cell. Biol. 20: 36263632.

Macaluso, M., Montanari, M., and Giordano, A. 2006. Rb family proteins as modulators of gene expression and new aspects regarding the interaction with chromatin remodeling enzymes. Oncogene 25: 5263-5267.

McClellan, K.A., Ruzhynsky, V.A., Douda, D.N., Vanderluit, J.L., Ferguson, K.L., Chen, D., Bremner, R., Park, D.S., Leone, G., and Slack, R.S. 2007. Unique requirement for Rb/E2F3 in neuronal migration: Evidence for cell cycle-independent functions. Mol. Cell. Biol. 27: 4825-4843.

Moon, N.S., and Dyson, N. 2008. E2F7 and E2F8 keep the E2F family in balance. Dev. Cell 14: 1-3.

Novitch, B.G., Mulligan, G.J., Jacks, T., and Lassar, A.B. 1996. Skeletal muscle cells lacking the retinoblastoma protein display defects in muscle gene expression and accumulate in S and G2 phases of the cell cycle. J. Cell Biol. 135: 441456.

Novitch, B.G., Spicer, D.B., Kim, P.S., Cheung, W.L., and Lassar, A.B. 1999. $\mathrm{pRb}$ is required for MEF2-dependent gene expression as well as cell-cycle arrest during skeletal muscle differentiation. Curr. Biol. 9: 449-459.

Pfaffl, M.W. 2001. A new mathematical model for relative quantification in real-time RT-PCR. Nucleic Acids Res. 29: e45.

Ren, B., Cam, H., Takahashi, Y., Volkert, T., Terragni, J., Young, R.A., and Dynlacht, B.D. 2002. E2F integrates cell cycle progression with DNA repair, replication, and G(2)/M checkpoints. Genes \& Dev. 16: 245-256.

Shan, B., Durfee, T., and Lee, W.H. 1996. Disruption of RB/E2F-1 interaction by single point mutations in E2F-1 enhances Sphase entry and apoptosis. Proc. Natl. Acad. Sci. 93: 679-684.

Sherr, C.J. 2004. Principles of tumor suppression. Cell 116: 235246.

Silva, J.M., Li, M.Z., Chang, K., Ge, W., Golding, M.C., Rickles, R.J., Siolas, D., Hu, G., Paddison, P.J., Schlabach, M.R., et al. 2005. Second-generation shRNA libraries covering the mouse and human genomes. Nat. Genet. 37: 1281-1288.

Stevens, C., and La Thangue, N.B. 2003. E2F and cell cycle control: A double-edged sword. Arch. Biochem. Biophys. 412: 157-169.

Tsai, S.Y., Opavsky, R., Sharma, N., Wu, L., Naidu, S., Nolan, E., Feria-Arias, E., Timmers, C., Opavska, J., de Bruin, A., et al. 2008. Mouse development with a single E2F activator. Nature 454: 1137-1141.

Tsang, W.Y., Wang, L., Chen, Z., Sanchez, I., and Dynlacht, B.D. 2007. SCAPER, a novel cyclin A-interacting protein that regulates cell cycle progression. J. Cell Biol. 178: 621-633. 
van Oevelen, C., Wang, J., Asp, P., Yan, Q., Kaelin Jr., W.G., Kluger, Y., and Dynlacht, B.D. 2008. A role for mammalian Sin3 in permanent gene silencing. Mol. Cell 32: 359-370.

Wenzel, P.L., Wu, L., de Bruin, A., Chong, J.L., Chen, W.Y., Dureska, G., Sites, E., Pan, T., Sharma, A., Huang, K., et al. 2007. $\mathrm{Rb}$ is critical in a mammalian tissue stem cell population. Genes \& Dev. 21: 85-97.

Wu, L., Timmers, C., Maiti, B., Saavedra, H.I., Sang, L., Chong, G.T., Nuckolls, F., Giangrande, P., Wright, F.A., Field, S.J., et al. 2001. The E2F1-3 transcription factors are essential for cellular proliferation. Nature 414: 457-462.

Wu, L., de Bruin, A., Saavedra, H.I., Starovic, M., Trimboli, A., Yang, Y., Opavska, J., Wilson, P., Thompson, J.C., Ostrowski, M.C., et al. 2003. Extra-embryonic function of $\mathrm{Rb}$ is essential for embryonic development and viability. Nature 421: 942947.

Zhu, L. 2005. Tumour suppressor retinoblastoma protein Rb: A transcriptional regulator. Eur. J. Cancer 41: 2415-2427. 


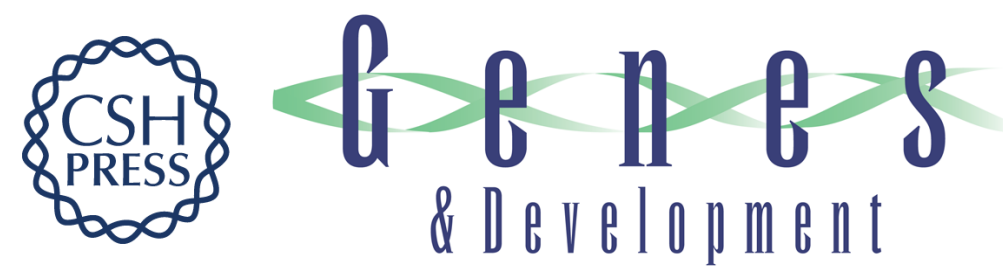

\section{E2f3b plays an essential role in myogenic differentiation through isoform-specific gene regulation}

Patrik Asp, Diego Acosta-Alvear, Mary Tsikitis, et al.

Genes Dev. 2009, 23:

Access the most recent version at doi:10.1101/gad.1727309

\section{Supplemental http://genesdev.cshlp.org/content/suppl/2009/01/07/23.1.37.DC1 Material}

References This article cites 40 articles, 16 of which can be accessed free at: http://genesdev.cshlp.org/content/23/1/37.full.html\#ref-list-1

\section{License}

Email Alerting

Receive free email alerts when new articles cite this article - sign up in the box at the top Service

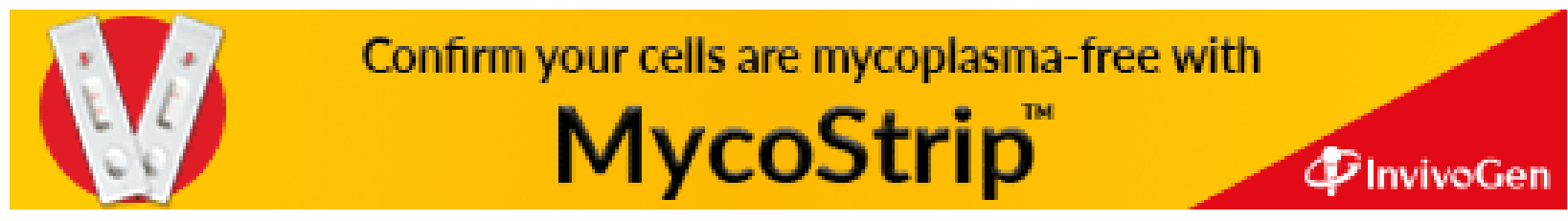

